\title{
Tracking the movement of eigenvalues via a corresponding Evans function
}

\author{
Benjamin Lewis * \\ Department of Mathematics and Statistics \\ Calvin College \\ Grand Rapids, MI 49546 \\ Faculty advisor: Prof. Todd Kapitula ${ }^{\dagger}$ \\ Department of Mathematics and Statistics \\ Calvin College \\ Grand Rapids, MI 49546
}

January 4, 2012

\begin{abstract}
In this paper we define the Evans function for Sturm-Liouville problems. We show that the Evans function is analytic in the spectral parameter, has zeros in one-to-one correspondence with the eigenvalues, and is under certain conditions what we call conjugate symmetric. We conclude by showing that the Evans function can be used to track the movement of the eigenvalues as the coefficients in the Sturm-Liouville problem are perturbed.
\end{abstract}

\section{Contents}

1. Introduction

2. The Evans Function 2

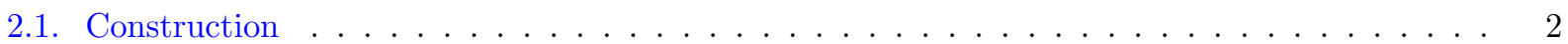

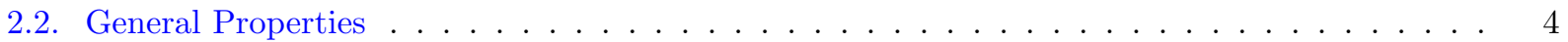

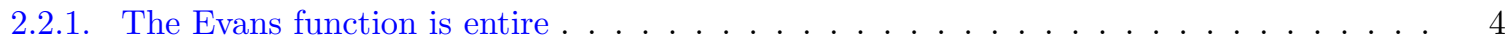

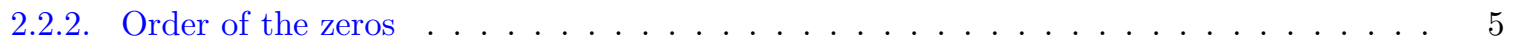

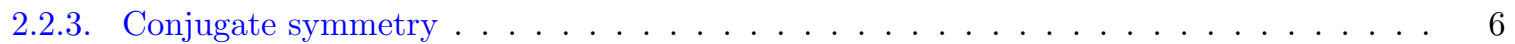

3. Application: tracking the movement of zeros 8

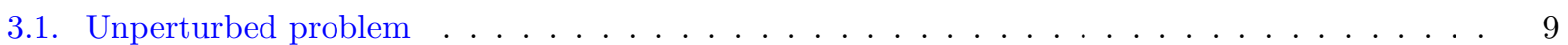

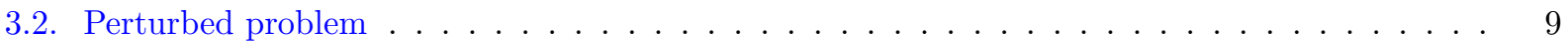

4. Further perturbative results 12

$\begin{array}{ll}\text { References } & 15\end{array}$

${ }^{*}$ E-mail: bdl5@students.calvin.edu

${ }^{\dagger}$ E-mail: tmk5@calvin.edu 


\section{INTRODUCTION}

In this paper we will study the following Sturm-Liouville problem for $-L \leq x \leq L$ :

$$
\begin{aligned}
& -\frac{\partial}{\partial x}\left(p(x) \frac{\partial y}{\partial x}\right)+q(x) y=\lambda w(x) y \\
& a_{1} y(-L)+b_{1} \frac{\partial y}{\partial x}(-L)=0, \quad a_{2} y(L)+b_{2} \frac{\partial y}{\partial x}(L)=0 .
\end{aligned}
$$

The goal in solving the Sturm-Liouville problem is to find values $\lambda$, the eigenvalues, for which there is a nontrivial solution to (1.1). A nontrivial solution associated with an eigenvalue is known as an eigenfunction. In (1.1) the constants $a_{j}, b_{j}$ are real-valued for $j=1,2$, and for normalization purposes satisfy

$$
a_{1}^{2}+b_{1}^{2}=a_{2}^{2}+b_{2}^{2}=1 .
$$

The real-valued coefficient $p(x)>0$ is assumed to be smooth, and the (possibly complex-valued) coefficients $q(x)$ and $w(x)$ are assumed to be piecewise smooth. In the context most familiar to undergraduate students the Sturm-Liouville problem appears after using the method of separation of variables when solving the heat equation, the wave equation, and Laplace's equation [2]. In the context of, e.g., the heat equation for a one-dimensional rod, the coefficient $p(x)$ represents the thermal conductivity of the material, $w(x)$ is proportional to the product of the specific heat and the mass density, and the term $q(x) y(x)$ arises under the assumption that the rod is not laterally insulated. An interesting physical application of (1.1) related to the study of optical solitons is discussed in [4].

In this paper we will derive a function, called the Evans function, which is analytic in the complex-valued spectral parameter $\lambda$, and which has the property that its zeros precisely correspond to the eigenvalues. In order to motivate the use of an Evans function consider a characteristic polynomial of a matrix. From a complex function perspective the characteristic polynomial can be thought of as a finite Taylor series. Finding the zeros of the characteristic polynomial is a common method used to find the eigenvalues of a matrix. These eigenvalues have algebraic multiplicity corresponding to the multiplicity of the zero of the characteristic polynomial. In the same way that the characteristic polynomial helps us find the eigenvalues of a matrix, we define an Evans function to be a function whose zeros correspond one-to-one to the eigenvalues of the Sturm-Liouville problem. Although we will not show this, it is the case that the order of each zero is equal to the algebraic multiplicity of the eigenvalue [1]. However, where the characteristic polynomial for a matrix is a finite Taylor polynomial, the Evans function is an infinite Taylor series. Thus, where the characteristic polynomial will have a finite number of zeros, it is necessarily the case that the Evans function will have countably infinitely many zeros.

The paper is ordered in the following manner. In Section 2 we construct the Evans function and give some of its properties. We start with the proof of analyticity, then prove all zeros are simple, and finally show that under specific conditions the Evans function is conjugate symmetric. In Section 3 we show how the Evans function can be used to track the eigenvalues of the Sturm-Liouville problem when the coefficients are varied. Finally, in Section 4 we use the Evans function and the Implicit Function Theorem to show how the eigenvalues move under small perturbations of the coefficients.

Acknowledgments. This work was supported in part by the National Science Foundation under grant number DMS-0806636. I would also like to acknowledge my research adviser, Prof. Todd Kapitula, for all his help and patience.

\section{The Evans FunCtion}

\subsection{Construction}

In order to solve the Sturm-Liouville problem we will first write down an equivalent vector-valued formulation. This new formulation will allow us to view the problem from a more geometric perspective. We 
begin by making the following substitution:

$$
u(x)=y(x), \quad v(x)=p(x) \frac{\partial y}{\partial x}(x)
$$

Note that in these variables the boundary conditions in (1.1) become

$$
a_{1} u(-L)+\frac{b_{1}}{p(-L)} v(-L)=0, \quad a_{2} u(L)+\frac{b_{2}}{p(L)} v(L)=0 .
$$

The manner in which we have defined $u$ and $v$ yields that the equivalent matrix system to (1.1) is

$$
\frac{\partial}{\partial x}\left(\begin{array}{l}
u \\
v
\end{array}\right)=\underbrace{\left(\begin{array}{cc}
0 & 1 / p \\
q-\lambda w & 0
\end{array}\right)}_{\boldsymbol{A}(x ; \lambda)}\left(\begin{array}{l}
u \\
v
\end{array}\right) .
$$

Using (2.2), the boundary conditions associated with solutions to the system (2.3) become

$$
\left(\begin{array}{l}
u \\
v
\end{array}\right)(-L) \propto\left(\begin{array}{c}
-b_{1} / p(-L) \\
a_{1}
\end{array}\right), \quad\left(\begin{array}{l}
u \\
v
\end{array}\right)(L) \propto\left(\begin{array}{c}
-b_{2} / p(L) \\
a_{2}
\end{array}\right) .
$$

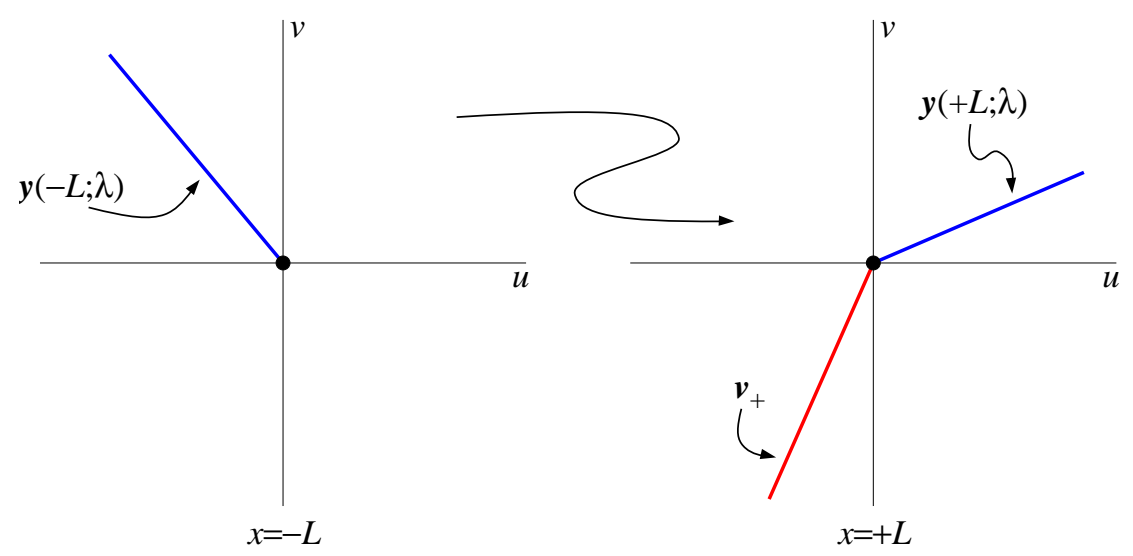

Figure 1: (color online) A cartoon of solution behavior for (2.3). The solution with the correct boundary condition at $x=-L$ is denoted by $\boldsymbol{y}(x ; \lambda)=\boldsymbol{\Phi}(x ; \lambda)(1,0)^{\mathrm{T}}$. The vector $\boldsymbol{v}_{+}=$ $\left(-b_{2} / p(L), a_{2}\right)^{\mathrm{T}}$ is the boundary condition at $x=+L$. In the pictured scenario $\boldsymbol{y}(+L ; \lambda) \not \propto \boldsymbol{v}_{+}$, so that $\lambda$ is not an eigenvalue.

In order to find the eigenvalues we will solve the differential equation with the initial condition being the boundary condition at $x=-L$, and then see how close the solution is to meeting the boundary condition at $x=L$. For this measure of proximity we use the determinant: this is what we will call the Evans function. If the solution satisfies the boundary conditions at both $x= \pm L$, then the determinant will be zero. Let

$$
\boldsymbol{\Phi}(x ; \lambda)=\left(\begin{array}{cc}
u(x ; \lambda) & \tilde{u}(x ; \lambda) \\
v(x ; \lambda) & \tilde{v}(x ; \lambda)
\end{array}\right)
$$

be a fundamental matrix solution to (2.3) with the "correct" initial condition at $x=-L$. In other words, $\boldsymbol{\Phi}(x ; \lambda)$ is defined via

$$
\frac{\partial}{\partial x} \boldsymbol{\Phi}=\boldsymbol{A}(x ; \lambda) \boldsymbol{\Phi}, \quad \boldsymbol{\Phi}(-L ; \lambda)=\left(\begin{array}{cc}
-b_{1} / p(-L) & c a_{1} \\
a_{1} & c b_{1} / p(-L)
\end{array}\right),
$$


where

$$
c=-\frac{1}{\left(b_{1} / p(-L)\right)^{2}+a_{1}^{2}}<0
$$

is the non-zero constant such that $\operatorname{det}(\boldsymbol{\Phi}(-L ; \lambda))=1$. Observe that since trace $(\boldsymbol{A}(x ; \lambda)) \equiv 0$, by Abel's formula $\operatorname{det}(\boldsymbol{\Phi}(x ; \lambda)) \equiv 1$ for all $x[\mathbf{5}]$. Now, the first column of $\boldsymbol{\Phi}(-L ; \lambda)$ is precisely the boundary condition at $x=-L$ (see (2.4)); thus, the solution to (2.3) given by

$$
\left(\begin{array}{l}
u \\
v
\end{array}\right)(x ; \lambda)=\boldsymbol{\Phi}(x ; \lambda)\left(\begin{array}{l}
1 \\
0
\end{array}\right)
$$

has the correct boundary condition at $x=-L$. If this solution has the correct boundary condition at $x=L$, then $\lambda$ will be an eigenvalue (see Figure 1). This observation brings us to our definition of the Evans function:

Definition 2.1. The Evans function is given by

$$
E(\lambda)=\operatorname{det}\left(\begin{array}{c}
\boldsymbol{\Phi}(L ; \lambda)\left(\begin{array}{c}
1 \\
0
\end{array}\right) \\
-b_{2} / p(L) \\
a_{2}
\end{array}\right)
$$

where the fundamental matrix solution $\boldsymbol{\Phi}(x ; \lambda)$ solves $(2.5)$.

Now we verify that our definition of the Evans function is consistent with the property that its zeros correspond to eigenvalues. If $\lambda=\alpha$ is a zero of $E(\lambda)$, then by the definition of the determinant it must be true that

$$
\boldsymbol{\Phi}(L ; \alpha)\left(\begin{array}{l}
1 \\
0
\end{array}\right)=\hat{c}\left(\begin{array}{c}
-b_{2} / p(L) \\
a_{2}
\end{array}\right), \quad \hat{c} \neq 0 .
$$

The right-hand side is the vector parallel to the boundary condition at $x=L$ (see (2.4)); consequently, $\lambda=\alpha$ is an eigenvalue. The converse is easy to see as well. Conversely, if $\lambda=\alpha$ is an eigenvalue then the solution of the differential equation solving from the left boundary conditions must satisfy the right, thus making the determinant zero.

\subsection{General Properties}

With our definition of the Evans function we can show some simple properties that will be useful for later analysis of the Sturm-Liouville problem.

\subsubsection{The Evans function is entire}

From complex analysis we know that a function is analytic in $\lambda$ if its partial derivative with respect to $\bar{\lambda}$ is zero [3]. This is simply another statement of the Cauchy-Riemann equations. We will now show that the Evans function has this property.

Theorem 2.2. The Evans function is entire, i.e., it is analytic for all $\lambda \in \mathbb{C}$.

Proof: We will show that the partial derivative with respect to $\bar{\lambda}$ of the Evans function will always be zero. Since the $\lambda$-dependence of the Evans function comes solely through the fundamental matrix solution $\mathbf{\Phi}(x ; \lambda)$, we have

$$
\frac{\partial}{\partial \bar{\lambda}} E(\lambda)=\operatorname{det}\left(\begin{array}{cc}
\frac{\partial}{\partial \bar{\lambda}} \boldsymbol{\Phi}(L ; \lambda)\left(\begin{array}{l}
1 \\
0
\end{array}\right) & -b_{2} / p(L) \\
a_{2}
\end{array}\right) .
$$

Thus, our goal will be accomplished if we can show that

$$
\frac{\partial}{\partial \bar{\lambda}} \boldsymbol{\Phi}(L ; \lambda)=\boldsymbol{O}_{2},
$$

where $\boldsymbol{O}_{2}$ is the $2 \times 2$ zero matrix. Now, from (2.5) we see that

$$
\frac{\partial}{\partial \bar{\lambda}}\left(\frac{\partial}{\partial x} \boldsymbol{\Phi}\right)=\frac{\partial}{\partial \bar{\lambda}}(\boldsymbol{A} \boldsymbol{\Phi})=\underbrace{\frac{\partial \boldsymbol{A}}{\partial \bar{\lambda}}}_{\boldsymbol{O}_{2}} \Phi+\boldsymbol{A} \frac{\partial \boldsymbol{\Phi}}{\partial \bar{\lambda}}, \quad \frac{\partial}{\partial \bar{\lambda}} \boldsymbol{\Phi}(-L ; \lambda)=\boldsymbol{O}_{2} .
$$


In other words, by letting

$$
\boldsymbol{\Psi}(x)=\frac{\partial}{\partial \bar{\lambda}} \boldsymbol{\Phi},
$$

and reversing the order of differentiation we have that $\boldsymbol{\Psi}$ solves the system

$$
\frac{\partial}{\partial x} \boldsymbol{\Psi}=\boldsymbol{A}(x ; \lambda) \boldsymbol{\Psi}, \quad \boldsymbol{\Psi}(-L)=\boldsymbol{O}_{2} .
$$

The solution to this system is $\boldsymbol{\Psi}(x) \equiv \boldsymbol{O}_{2}$, which in particular implies that

$$
\boldsymbol{\Psi}(L)=\boldsymbol{O}_{2} \quad \Rightarrow \quad \frac{\partial}{\partial \bar{\lambda}} \boldsymbol{\Phi}(L ; \lambda)=\boldsymbol{O}_{2} .
$$

This is the desired result.

\subsubsection{Order of the zeros}

Since the Evans function is entire, every zero of the Evans function has a finite order $k$, which at a zero $\lambda=\alpha$ is defined by

$$
E(\alpha)=\frac{\partial E}{\partial \lambda}(\alpha)=\cdots=\frac{\partial^{k-1} E}{\partial \lambda^{k-1}}(\alpha)=0, \quad \frac{\partial^{k} E}{\partial \lambda^{k}}(\alpha) \neq 0 .
$$

A simple zero is a zero with $k=1$, i.e., the function is zero with a nonzero derivative. We now demonstrate that for the classical Sturm-Liouville problem with real-valued coefficients there is an equivalence between the order of a zero and the multiplicity of the eigenvalue. We will implicitly use the fact that for real-valued coefficients with $p, w>0$, then all of the eigenvalues for the Sturm-Liouville problem, and consequently all of the zeros of the Evans function, are necessarily real-valued [2].

thm:simple Theorem 2.3 (Simple Zeros). If $p, q, w$ are real-valued with $p, w>0$, then all real-valued zeros of the Evans function will be simple.

Proof: In order to show that all zeros are simple zeros under the hypothesis we will show that at a zero the derivative of the Evans function must be nonzero. Taking the derivative with respect to $\lambda$ in (2.5) and using the product rule results in the system

$$
\frac{\partial}{\partial \lambda}\left(\frac{\partial}{\partial x} \boldsymbol{\Phi}\right)=\frac{\partial \boldsymbol{A}}{\partial \lambda} \boldsymbol{\Phi}+\boldsymbol{A} \frac{\partial \boldsymbol{\Phi}}{\partial \lambda}, \quad \frac{\partial}{\partial \lambda} \boldsymbol{\Phi}(-L ; \lambda)=\boldsymbol{O}_{2} .
$$

Let

$$
\boldsymbol{\Psi}=\frac{\partial}{\partial \lambda} \boldsymbol{\Phi}
$$

so that upon reversing the order of differentiation (2.7) is equivalent to:

$$
\frac{\partial}{\partial x} \boldsymbol{\Psi}=\boldsymbol{A}(x ; \lambda) \boldsymbol{\Psi}+\frac{\partial \boldsymbol{A}}{\partial \lambda} \boldsymbol{\Phi}, \quad \boldsymbol{\Psi}(-L ; \lambda)=\boldsymbol{O}_{2} .
$$

We now apply variation of parameters in order to solve the above system to get

$$
\boldsymbol{\Psi}(x ; \lambda)=\boldsymbol{\Phi}(x ; \lambda) \int_{-L}^{x} \boldsymbol{\Phi}(s ; \lambda)^{-1} \frac{\partial \boldsymbol{A}}{\partial \lambda} \boldsymbol{\Phi}(s ; \lambda) \mathrm{d} s .
$$

Since

$$
\boldsymbol{\Phi}=\left(\begin{array}{cc}
u & \tilde{u} \\
v & \tilde{v}
\end{array}\right) \Rightarrow \boldsymbol{\Phi}^{-1}=\left(\begin{array}{rr}
\tilde{v} & -\tilde{u} \\
-v & u
\end{array}\right),
$$

(we use the fact that $\operatorname{det}(\boldsymbol{\Phi}) \equiv 1$ ), upon substituting we find

$$
\begin{aligned}
\boldsymbol{\Psi}(L ; \lambda)\left(\begin{array}{c}
1 \\
0
\end{array}\right) & =\boldsymbol{\Phi}(L ; \lambda) \int_{-L}^{L} \boldsymbol{\Phi}(s ; \lambda)^{-1} \frac{\partial \boldsymbol{A}}{\partial \lambda} \boldsymbol{\Phi}(s ; \lambda) \mathrm{d} s\left(\begin{array}{c}
1 \\
0
\end{array}\right) \\
& =\boldsymbol{\Phi}(L ; \lambda) \int_{-L}^{L}\left(\begin{array}{c}
w(s) u(s) \tilde{u}(s) \\
-w(s) u(s)^{2}
\end{array}\right) \mathrm{d} s .
\end{aligned}
$$


If $\lambda=\lambda_{0}$ is an eigenvalue, so that $E\left(\lambda_{0}\right)=0$, then it is necessarily the case that

$$
\boldsymbol{\Phi}\left(L ; \lambda_{0}\right)\left(\begin{array}{l}
1 \\
0
\end{array}\right)=\hat{c}\left(\begin{array}{c}
-b_{2} / p(L) \\
a_{2}
\end{array}\right), \quad \hat{c} \neq 0
$$

Applying the product rule gives

$$
\begin{aligned}
\frac{\partial E}{\partial \lambda}\left(\lambda_{0}\right) & =\operatorname{det}\left(\frac{\partial}{\partial \lambda} \boldsymbol{\Phi}\left(L ; \lambda_{0}\right)\left(\begin{array}{c}
1 \\
0
\end{array}\right)\right. \\
& =\frac{1}{\hat{c}} \operatorname{det}\left(\boldsymbol{\Psi}\left(L ; \lambda_{0}\right)\left(\begin{array}{c}
1 \\
0
\end{array}\right) \boldsymbol{\Phi}\left(L ; \lambda_{0}\right)\left(\begin{array}{c}
1 \\
0
\end{array}\right)\right) \\
& =\frac{1}{\hat{c}} \operatorname{det}\left(\boldsymbol{\Phi}\left(L ; \lambda_{0}\right)\right) \operatorname{det}\left(\begin{array}{cc}
\int_{-L}^{L} w(s) u(s) \tilde{u}(s) \mathrm{d} s & 1 \\
-\int_{-L}^{L} w(s) u(s)^{2} \mathrm{~d} s & 0
\end{array}\right) \\
& =\frac{1}{\hat{c}} \int_{-L}^{L} w(s) u(s)^{2} \mathrm{~d} s .
\end{aligned}
$$

Since $w(x)>0$ and $u(x) \not \equiv 0$, and since $u$ is real-valued because $p, q, w$ are real-valued, we can then conclude that the first derivative is nonzero.

Remark 2.4. We have shown in detail that for $p, q, w$ real-valued the order of a zero of the Evans function is equal to the multiplicity of the eigenvalue. This result is true in general, i.e., the order of a zero of the Evans function is equal to the (algebraic) multiplicity of the eigenvalue [1].

Remark 2.5. If $\operatorname{Re}\left(u_{1}(x)\right) \not \equiv 0$, which can occur if $q$ and/or $w$ are not real-valued, then it is possible for the first derivative to be zero, so that the zero of the Evans function is not simple. We will consider this possibility later in the paper.

\subsubsection{Conjugate symmetry}

We call an analytic function $f$ that has the property $f(\alpha)=\overline{f(\bar{\alpha})}$ conjugate symmetric. An important property of conjugate symmetric functions is that the zeros come in complex-conjugate pairs. We find that under specific conditions the Evans function is conjugate symmetric, which implies that all of the eigenvalues of the Sturm-Liouville problem will come in complex-conjugate pairs.

Lemma 2.6. If $p, q, w$ are real-valued, then the Evans function is conjugate symmetric regardless of boundary conditions.

Proof: Recall that (2.5) is given by

$$
\frac{\partial}{\partial x} \boldsymbol{\Phi}=\boldsymbol{A}(x ; \lambda) \mathbf{\Phi}, \quad \boldsymbol{\Phi}(-L ; \lambda)=\left(\begin{array}{cc}
-b_{1} / p(-L) & c a_{1} \\
a_{1} & c b_{1} / p(-L)
\end{array}\right) .
$$

Taking the complex-conjugate gives

$$
\frac{\partial}{\partial x} \overline{\mathbf{\Phi}(x ; \lambda)}=\overline{\boldsymbol{A}(x ; \lambda) \boldsymbol{\Phi}(x ; \lambda)}, \quad \overline{\mathbf{\Phi}(-L ; \lambda)}=\left(\begin{array}{cc}
-b_{1} / p(-L) & c a_{1} \\
a_{1} & c b_{1} / p(-L)
\end{array}\right)
$$

(here we use the fact that the coefficients are real-valued). Since

$$
\overline{\boldsymbol{A}(x ; \lambda)}=\left(\begin{array}{cc}
0 & \overline{1 / p} \\
\overline{q-\lambda w} & 0
\end{array}\right)=\left(\begin{array}{cc}
0 & 1 / p \\
q-\bar{\lambda} w & 0
\end{array}\right)=\boldsymbol{A}(x ; \bar{\lambda})
$$

the conjugated differential equation becomes

$$
\frac{\partial}{\partial x} \overline{\mathbf{\Phi}(x ; \lambda)}=\boldsymbol{A}(x ; \bar{\lambda}) \overline{\mathbf{\Phi}(x ; \lambda)}, \quad \overline{\mathbf{\Phi}(-L ; \lambda)}=\left(\begin{array}{cc}
-b_{1} / p(-L) & c a_{1} \\
a_{1} & c b_{1} / p(-L)
\end{array}\right) .
$$


Thus, by the uniqueness of solutions to initial-value problems for ODEs it must be the case that $\overline{\mathbf{\Phi}(x ; \lambda)}=$ $\boldsymbol{\Phi}(x ; \bar{\lambda})$. As a consequence we then conclude that

$$
\begin{aligned}
& E(\bar{\lambda})=\operatorname{det}\left(\begin{array}{cc}
\boldsymbol{\Phi}(L ; \bar{\lambda})\left(\begin{array}{c}
1 \\
0
\end{array}\right) & -b_{2} / p(L) \\
a_{2}
\end{array}\right) \\
& =\operatorname{det}\left(\overline{\mathbf{\Phi}(L ; \lambda)}\left(\begin{array}{l}
1 \\
0
\end{array}\right) \begin{array}{c}
-b_{2} / p(L) \\
a_{2}
\end{array}\right) \\
& \left.=\overline{\operatorname{det}\left(\boldsymbol{\Phi}(L ; \lambda)\left(\begin{array}{l}
1 \\
0
\end{array}\right)\right.} \begin{array}{c}
-b_{2} / p(L) \\
a_{2}
\end{array}\right) \\
& =\overline{E(\lambda)} \text {. }
\end{aligned}
$$

If $p, q, w$ are not real-valued, then we need to put some conditions on both these functions and the boundary conditions in order to retain the feature that the Evans function be conjugate symmetric. We start with a preliminary result regarding solutions to the original Sturm-Liouville problem.

lem:sym Lemma 2.7. Consider the Sturm-Liouville ODE

$$
-\frac{\partial}{\partial x}\left(p(x) \frac{\partial y}{\partial x}\right)+q(x) y=\lambda w(x) y
$$

Suppose that $p(x)>0$ is even and real-valued, and further suppose that

$$
\overline{q(x)}=q(-x), \quad \overline{w(x)}=w(-x) .
$$

Denote a solution to the $O D E \underline{\text { as } y(x ; \lambda)}$. If for a given $\lambda=\alpha \in \mathbb{C}$ the ODE has the solution $y(x ; \alpha)$, then for $\lambda=\bar{\alpha}$ there is the solution $\overline{y(-x ; \bar{\alpha})}$.

Proof: Taking the complex-conjugate of the ODE yields

$$
-\frac{\partial}{\partial x}\left(\overline{p(x)} \frac{\partial \bar{y}}{\partial x}\right)+\overline{q(x)} \bar{y}=\bar{\lambda} \overline{w(x)} \bar{y}
$$

and using the assumption on $q$ and $w$, and the fact that $p$ is real-valued, gives

$$
-\frac{\partial}{\partial x}\left(p(x) \frac{\partial \bar{y}}{\partial x}\right)+q(-x) \bar{y}=\bar{\lambda} w(-x) \bar{y}
$$

Letting $t=-x$ and $z(t)=\overline{y(t)}$, and using the fact that $p$ is even, leads to the ODE

$$
-\frac{\partial}{\partial t}\left(p(t) \frac{\partial z}{\partial t}\right)+q(t) z=\bar{\lambda} w(t) z
$$

Since this is the same equation except for the mapping $\lambda \mapsto \bar{\lambda}$, the result is now proved.

With the result of Lemma 2.7 in hand we are now able to show that the Evans function will continue to be conjugate symmetric even for complex-valued coefficients.

t:11 Theorem 2.8. Regarding the coefficients for the Sturm-Liouville problem suppose that $p(x)>0$ is realvalued and even, and further suppose that

$$
\overline{q(x)}=q(-x), \quad \overline{w(x)}=w(-x) .
$$

Regarding the boundary conditions, suppose that the coefficients satisfy

$$
a_{1}=a_{2}, \quad b_{1}=b_{2}
$$

in other words, the boundary conditions are the same at $x= \pm L$. The Evans function is then conjugate symmetric, i.e., $E(\lambda)=\overline{E(\bar{\lambda})}$. 
Proof: It is equivalent to show that $E(\lambda)=\overline{E(\bar{\lambda})}$. Using the fact that $a_{1}=a_{2}, b_{1}=b_{2}$ allows us to say

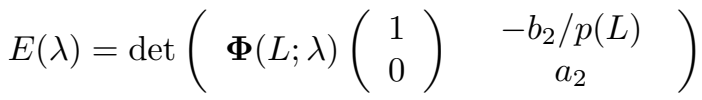

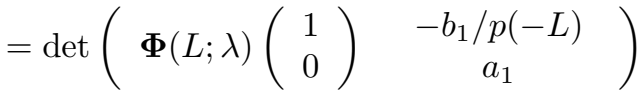

$$
\begin{aligned}
& =\operatorname{det}\left(\boldsymbol{\Phi}(L ; \lambda)\left(\begin{array}{l}
1 \\
0
\end{array}\right) \quad \boldsymbol{\Phi}(-L ; \lambda)\left(\begin{array}{l}
1 \\
0
\end{array}\right)\right) .
\end{aligned}
$$

Recall from the transformation (2.1) that solutions to the system (2.3) satisfy

$$
\left(\begin{array}{l}
u \\
v
\end{array}\right)(x ; \lambda)=\left(\begin{array}{c}
y \\
p(x) \partial y / \partial x
\end{array}\right)(x ; \lambda) \text {. }
$$

Since

we can say that

$$
\boldsymbol{\Phi}(x ; \lambda)\left(\begin{array}{l}
1 \\
0
\end{array}\right)=\left(\begin{array}{l}
u \\
v
\end{array}\right)(x ; \lambda)=\left(\begin{array}{c}
y \\
p(x) \partial y / \partial x
\end{array}\right)(x ; \lambda)
$$

$$
E(\lambda)=\operatorname{det}\left(\begin{array}{cc}
y(L ; \lambda) & y(-L ; \lambda) \\
p(L) \partial y / \partial x(L ; \lambda) & p(-L) \partial y / \partial x(-L ; \lambda)
\end{array}\right) .
$$

As a consequence of Lemma 2.7 we know that solutions satisfy

$$
y(x ; \lambda)=\overline{y(-x ; \bar{\lambda})}, \quad \frac{\partial y}{\partial x}(x ; \alpha)=-\overline{\frac{\partial y}{\partial x}(-x ; \bar{\alpha})} .
$$

The fact that $p(x)$ is even, so that $p(-L)=p(L)$, finally allows us to write

$$
\begin{aligned}
& E(\lambda)=\operatorname{det}\left(\begin{array}{cc}
\overline{y(-L ; \bar{\lambda})} & \overline{y(L ; \bar{\lambda})} \\
-p(L) \overline{\partial y / \partial x(-L ; \bar{\lambda})} & -p(L) \overline{\partial y / \partial x(L ; \bar{\lambda})}
\end{array}\right)
\end{aligned}
$$

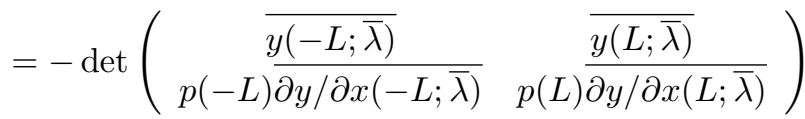

$$
\begin{aligned}
& =\operatorname{det}\left(\begin{array}{cc}
\overline{y(L ; \bar{\lambda})} & \overline{y(-L ; \bar{\lambda})} \\
p(L) \overline{\partial y / \partial x(L ; \bar{\lambda})} & p(-L) \overline{\partial y / \partial x(-L ; \bar{\lambda})}
\end{array}\right) \\
& =\operatorname{det}\left(\overline{\mathbf{\Phi}(L ; \bar{\lambda})}\left(\begin{array}{l}
1 \\
0
\end{array}\right) \overline{\mathbf{\Phi}(-L ; \bar{\lambda})}\left(\begin{array}{l}
1 \\
0
\end{array}\right)\right)
\end{aligned}
$$

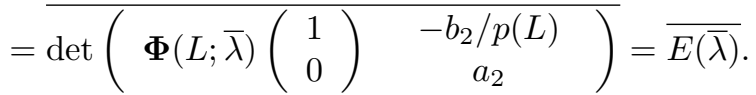

\section{Application: tracking the MOVEMENT OF ZEROS}

We now wish to use the results of the previous section in order to study the eigenvalue problem for a particular set of coefficients. For the coefficients we will let

$$
p(x)=w(x)=1, \quad q(x)=V(x),
$$

and and for the boundary conditions we will set

$$
a_{1}=a_{2}=1, \quad b_{1}=b_{2}=0 .
$$

Our Sturm-Liouville problem then becomes

$$
-\frac{\partial^{2} y}{\partial x^{2}}+V(x) y=\lambda y ; \quad y(-L)=0, y(L)=0 .
$$

We call $V(x)$ the potential. Notice when $V(x)$ is real valued, all of the zeros for the Evans function will be real and simple. However, if $\operatorname{Im}(V(x)) \neq 0$, then it is possible to have zeros with order greater than one, and zeros with non-zero imaginary part. 


\subsection{Unperturbed problem}

The system equivalent of the Sturm-Liouville problem is

$$
\frac{\partial}{\partial x} \boldsymbol{\Phi}=\underbrace{\left(\begin{array}{cc}
0 & 1 \\
V(x)-\lambda & 0
\end{array}\right)}_{\boldsymbol{A}(x ; \lambda)} \boldsymbol{\Phi}, \quad \boldsymbol{\Phi}(-L ; \lambda)=\left(\begin{array}{rr}
0 & -1 \\
1 & 0
\end{array}\right) .
$$

The system is easiest to solve when $V(x) \equiv 0$; indeed, the fundamental matrix solution is given by

$$
\boldsymbol{\Phi}(x ; \lambda)=\left(\begin{array}{cc}
\sin (\sqrt{\lambda}(x+L)) / \sqrt{\lambda} & -\cos (\sqrt{\lambda}(x+L)) \\
\cos (\sqrt{\lambda}(x+L)) & \sqrt{\lambda} \sin (\sqrt{\lambda}(x+L))
\end{array}\right) .
$$

Thus, for the Evans function we have

$$
E(\lambda)=\operatorname{det}\left(\boldsymbol{\Phi}(L ; \lambda)\left(\begin{array}{l}
1 \\
0
\end{array}\right) \quad \begin{array}{l}
0 \\
1
\end{array}\right)=\frac{\sin (2 L \sqrt{\lambda})}{\sqrt{\lambda}} .
$$

Initially, it appears as if the Evans function has a branch point at $\lambda=0$, which contradicts the fact that the Evans function is entire. However, upon doing the Taylor expansion about $\lambda=0$ we get

$$
E(\lambda)=\frac{\sum_{n=0}^{\infty} \frac{(-1)^{n}}{(2 n+1) !}(2 L \sqrt{\lambda})^{2 n+1}}{\sqrt{\lambda}}=\sum_{n=0}^{\infty} \frac{(-1)^{n}(2 L)^{2 n+1}}{(2 n+1) !} \lambda^{n},
$$

which is a power series that converges for all $\lambda$. Regarding the zeros of the Evans function, it is clear that $E(0)=2 L>0$, and it is easy to see the simple real-valued zeros occur at

$$
\lambda=\frac{\pi^{2} n^{2}}{4 L^{2}}, \quad n=1,2, \ldots
$$

Now we would like to plot the Evans function; however, a complex-valued function is hard to graph in two-dimensions. In order to overcome this we use the idea of domain coloring. Write a complex number as

$$
z=|z| \mathrm{e}^{\mathrm{i} \theta}, \quad 0 \leq \theta<2 \pi .
$$

For each value of the argument of the complex number, $\theta$, we assign a unique color. In order to take into account the magnitude of the complex number, $|z|$, we assign to that color an intensity. It is often the case that the intensity is light for small magnitudes, and dark for large magnitudes. However, in all of our graphs we will ignore this component of a complex number, and instead just use the argument in order to locate the zeros of the Evans function. In particular, from the argument principle we know that a zero will correspond to a discontinuity in the argument of the analytic function, and using the winding number we know that the integer number of times each color appears in a small neighborhood of a zero (this is the same number for all of the colors) is the order of a zero. In other words, one can tell the multiplicity of the zero based on number of times a particular color goes around a zero.

We apply domain coloring to the Evans function $E(\lambda)$ of (3.2) with $L=\pi / 2$ in order to get Figure 2. The zeros of the Evans function are the points where all of the colors meet, which in Figure 2 are the points

$$
z \in\{1+0 \mathrm{i}, 4+0 \mathrm{i}, 9+0 \mathrm{i}, 16+0 \mathrm{i}, 25+0 \mathrm{i}, 36+0 \mathrm{i}, 49+0 \mathrm{i}\} .
$$

In Figure 2 we see that all the zeros are simple zeros, which is consistent with what we proved earlier.

\subsection{Perturbed problem}

We will now use Dirac delta functions to move the zeros of the Evans function of the unperturbed problem. Before we can do that, however, we need to know how to solve the Sturm-Liouville problem with a Dirac delta function potential. Using $V(x)=\eta \delta(x-p)$, write the system (3.1) as

$$
\frac{\partial u}{\partial x}=v, \quad \frac{\partial v}{\partial x}=(\eta \delta(x-a)-\lambda) u .
$$




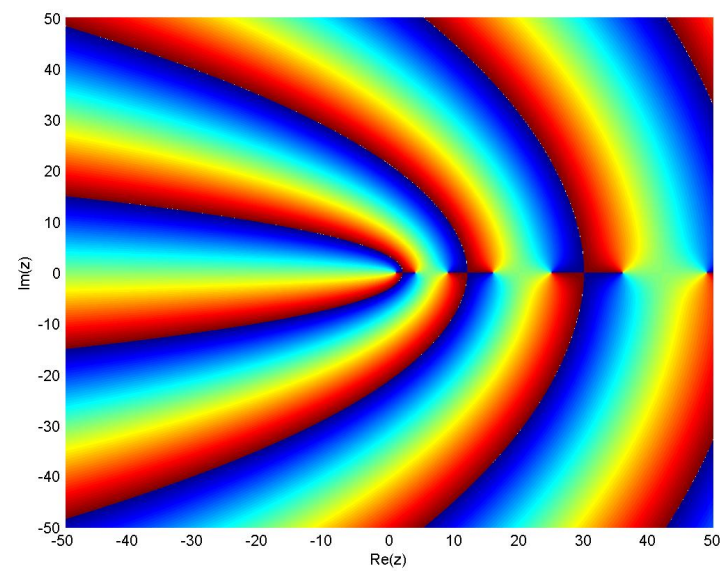

Figure 2: (color online) A domain coloring plot of the Evans function of (3.2) when $L=\pi / 2$.

We impose the condition that $u=y$ be continuous at $x=a$; however we do not require that $v=\partial y / \partial x$ be continuous at $x=a$. We must find how $v$ changes upon moving across $x=a$. For a generic function $f$ set

$$
f\left(a^{-}\right)=\lim _{x \rightarrow a^{-}} f(x), \quad f\left(a^{+}\right)=\lim _{x \rightarrow a^{+}} f(x) .
$$

Integrating the equation for $\partial v \partial x$ in (3.3) we get

$$
v(x)=\int_{-L}^{x}[\eta \delta(s-a)-\lambda] u(s) \mathrm{d} s= \begin{cases}-\lambda \int_{-L}^{x} u(s) \mathrm{d} s+v(-L), & x<a \\ \eta u(a)-\lambda \int_{-L}^{x} u(s) \mathrm{d} s+v(-L), & x>a .\end{cases}
$$

This yields that

$$
v\left(a^{-}\right)=-\lambda \int_{-L}^{a} u(s) \mathrm{d} s+v(-L)
$$

and

$$
v\left(a^{+}\right)=\eta u(a)-\lambda \int_{-L}^{a} u(s) \mathrm{d} s+v(-L)=\eta u(a)+v\left(a^{-}\right) .
$$

Thus, in matrix form the solutions must satisfy the jump condition

$$
\left(\begin{array}{l}
u \\
v
\end{array}\right)\left(a^{+}\right)=\underbrace{\left(\begin{array}{ll}
1 & 0 \\
\eta & 1
\end{array}\right)}_{\boldsymbol{J}(\eta)}\left(\begin{array}{l}
u \\
v
\end{array}\right)\left(a^{-}\right)
$$

We are now ready to solve (3.1) when $V(x)$ is a finite sum of delta functions. Set

$$
\boldsymbol{H}(x, a)=\left(\begin{array}{cc}
\cos (\sqrt{\lambda}(x-a)) & \sin (\sqrt{\lambda}(x-a)) / \sqrt{\lambda} \\
-\sqrt{\lambda} \sin (\sqrt{\lambda}(x-a)) & \cos (\sqrt{\lambda}(x-a))
\end{array}\right) .
$$

One has that $\boldsymbol{H}(x, a)$ solves the homogeneous differential equation, and $\boldsymbol{H}(a, a)=\boldsymbol{I}_{2}$, where $\boldsymbol{I}_{2}$ is $2 \times 2$ identity matrix. If we multiply $\boldsymbol{H}(x,-L)$ by the vector $\boldsymbol{e}_{2}=(0,1)^{\mathrm{T}}$, then we get a solution to the SturmLiouville problem which matches the boundary condition $y(-L)=0$. Suppose that

$$
V(x)=\eta\left[\delta\left(x+\rho_{\eta}\right)+\delta\left(x-\rho_{\eta}\right)\right], \quad 0<\rho_{\eta}<L .
$$


We then have that

$$
\boldsymbol{\Phi}(x ; \lambda)\left(\begin{array}{l}
1 \\
0
\end{array}\right)= \begin{cases}\boldsymbol{H}(x,-L) \boldsymbol{e}_{2}, & -L \leq x<-\rho_{\eta} \\
\boldsymbol{H}\left(x,-\rho_{\eta}\right) \boldsymbol{J}(\eta) \boldsymbol{H}\left(-\rho_{\eta},-L\right) \boldsymbol{e}_{2}, & -\rho_{\eta}<x<\rho_{\eta} \\
\boldsymbol{H}\left(x, \rho_{\eta}\right) \boldsymbol{J}(\eta) \boldsymbol{H}\left(\rho_{\eta},-\rho_{\eta}\right) \boldsymbol{J}(\eta) \boldsymbol{H}\left(-\rho_{\eta},-L\right) \boldsymbol{e}_{2}, & -\rho_{\eta}<x \leq L .\end{cases}
$$

In particular, we have that

$$
\boldsymbol{\Phi}(L ; \lambda)\left(\begin{array}{c}
1 \\
0
\end{array}\right)=\boldsymbol{H}\left(L, \rho_{\eta}\right) \boldsymbol{J}(\eta) \boldsymbol{H}\left(\rho_{\eta},-\rho_{\eta}\right) \boldsymbol{J}(\eta) \boldsymbol{H}\left(-\rho_{\eta},-L\right)\left(\begin{array}{c}
0 \\
1
\end{array}\right),
$$

which gives us an analytic expression for the Evans function of

$$
E(\lambda)=\operatorname{det}\left(\boldsymbol{\Phi}(L ; \lambda)\left(\begin{array}{l}
1 \\
0
\end{array}\right) \quad \begin{array}{l}
0 \\
1
\end{array}\right) .
$$

Since the potential is real-valued the zeros of the Evans functions will still be real and simple; however, depending upon the values of $\eta$ and $\rho_{\eta}$ they will move and possibly get close to each other. For large $\eta$ a Maple calculation shows that

$$
\frac{E(\lambda)}{\eta^{2}}=\frac{2 \sin \left(2 \rho_{\eta} \sqrt{\lambda}\right)+\sin \left(-4 \rho_{\eta} \sqrt{\lambda}+2 L \sqrt{\lambda}\right)-\sin (2 L \sqrt{\lambda})}{4 \lambda^{3 / 2}}+\mathcal{O}\left(\eta^{-1}\right) .
$$

We will fix a large $\eta$ : for our purposes we normally chose $\eta=20$. We then manipulate $\rho_{\eta}$ to move the zeros. Figure 3 is a plot of the differences between zeros when $L=\pi / 2$. It is clear that the position of the delta function, i.e., the value of $\rho_{\eta}$, has a significant effect on the distance between successive zeros.

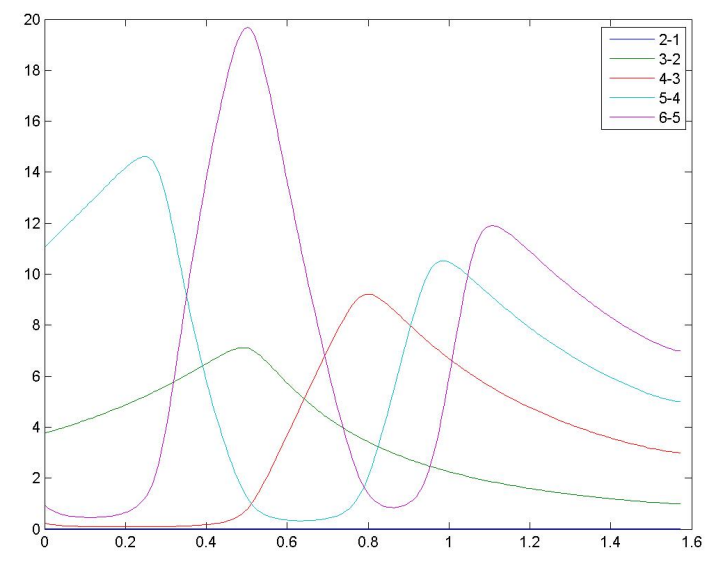

Figure 3: (color online) The distance between successive zeros of the Evans function given by (3.5) when $L=\pi / 2$. The vertical axis is the difference, and the horizontal axis is the value of $\rho_{\eta}$. The labeling " $j-k$ " means the difference between the $j^{\text {th }}$ and $k^{\text {th }}$ zeros. For example, $2-1$ is the difference between the second and first zeros. Note that 2-1 is always the smallest; however, the distance between the other zeros strongly depends on the value of $\rho_{\eta}$.

In order to allow the zeros to form double zeros and then move off the real axis we must add an imaginary part to the potential. However, because we want the Evans function to remain conjugate symmetric, we must choose the imaginary part of the potential with care. Because we have already done the work to understand the delta function we now consider the potential

$$
V(x)=\eta\left[\delta\left(x+\rho_{\eta}\right)+\delta\left(x-\rho_{\eta}\right)\right]+\mathrm{i} \sigma\left[-\delta\left(x+\rho_{\sigma}\right)+\delta\left(x-\rho_{\sigma}\right)\right] .
$$


Note that the imaginary part of the potential is odd in $x$, and the real part is even in $x$; hence, the hypothesis on the potential needed in Theorem 2.8 is satisfied. The Evans function will be conjugate symmetric, so that in order for a zero to have nonzero real part it must be the case that as $\sigma$ is increased a pair of simple real zeros first collides. The expectation is that the zeros which are closest when $\sigma=0$ will collide first. From Figure 3 we then expect that the first two zeros will collide first, and that zeros four and five will collide second. Figure 4 shows zeros splitting off the real axis for increasing $\sigma$ with $\rho_{\eta}=\rho_{\sigma}=L / 3 \sim 0.52$ (recall that $L=\pi / 2$ ). We see that our intuition is correct.

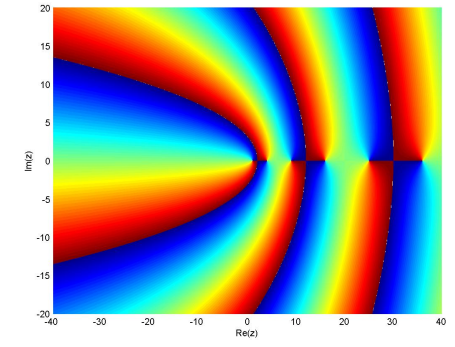

$\sigma=0$

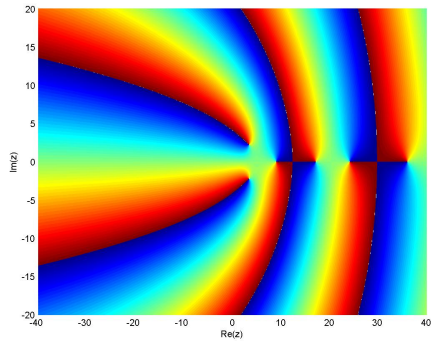

$\sigma=3$

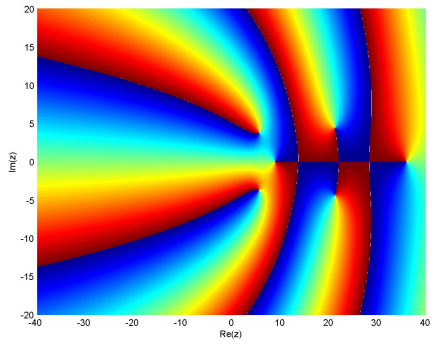

$\sigma=6$

Figure 4: (color online) The real zeros of the Evans function colliding and becoming complexvalued for the potential of the form (3.6). Here $\eta=20$, and the value of $\sigma$ is given under each plot.

\section{FurTher PERTURBATIVE RESUlts}

We now wish to show how the Evans function can be used to analytically track the movement of simple zeros under small perturbations of the coefficients in the Sturm-Liouville problem. Suppose that for the Sturm-Liouville problem (1.1) the functions $p, q, w$ depend smoothly upon a parameter, say $\epsilon$. We will assume that

$$
p=p_{0}+\epsilon p_{1}, \quad q=q_{0}+\epsilon q_{1}, \quad w=w_{0}+\epsilon w_{1} .
$$

If when $\epsilon=0, \lambda=\lambda_{0}$ is a simple eigenvalue, so that the Evans function, now written as $E(\lambda, \epsilon)$, satisfies $E_{\lambda}\left(\lambda_{0}, 0\right) \neq 0$, where for the sake of clarity of exposition we are using the notation

$$
E_{\lambda}=\frac{\partial E}{\partial \lambda}, \quad E_{\epsilon}=\frac{\partial E}{\partial \epsilon},
$$

then by the Implicit Function Theorem we know that there is a smooth curve $\lambda(\epsilon)$ with $\lambda(0)=\lambda_{0}$ such that $E(\lambda(\epsilon), \epsilon) \equiv 0$. The curve has the Taylor expansion given by

$$
\lambda(\epsilon)=\lambda_{0}+\lambda_{\epsilon}(0) \epsilon+\mathcal{O}\left(\epsilon^{2}\right), \quad \lambda_{\epsilon}(0)=-\frac{E_{\epsilon}\left(\lambda_{0}, 0\right)}{E_{\lambda}\left(\lambda_{0}, 0\right)} .
$$

If $y_{0}$ is the eigenfunction associated with $\lambda_{0}$, then we have seen in the proof of the Simple Zeros Theorem 2.3 that

$$
E_{\lambda}\left(\lambda_{0}, 0\right)=\frac{1}{\hat{c}} \int_{-L}^{L} w_{0}(s) y_{0}(s)^{2} \mathrm{~d} s,
$$

where the nonzero constant $\hat{c}$ is defined via

$$
\boldsymbol{\Phi}\left(L ; \lambda_{0}, 0\right)\left(\begin{array}{l}
1 \\
0
\end{array}\right)=\hat{c}\left(\begin{array}{c}
-b_{2} / p_{0}(L) \\
a_{2}
\end{array}\right) .
$$

In (4.4) we are referring to the fundamental matrix solution $\boldsymbol{\Phi}(x ; \lambda, \epsilon)$ corresponding to system (2.5), where now $\boldsymbol{A}=\boldsymbol{A}(x ; \lambda, \epsilon)$ is given in (2.3). In other words, upon using the fact that $v=p \partial y / \partial x$ (see (2.1)), when 
$\epsilon=0$ the eigenfunction satisfies

$$
y_{0}(-L)=-\frac{b_{1}}{p_{0}(-L)}, \frac{\partial y_{0}}{\partial x}(-L)=\frac{a_{1}}{p_{0}(-L)} ; \quad y_{0}(L)=-\hat{c} \frac{b_{2}}{p_{0}(L)}, \frac{\partial y_{0}}{\partial x}(L)=\hat{c} \frac{a_{2}}{p_{0}(L)} .
$$

In order to understand the movement of the simple zero it is only necessary to compute $E_{\epsilon}\left(\lambda_{0}, 0\right)$.

lem:41 Lemma 4.1. Suppose that the coefficients for the Sturm-Liouville problem are given by (4.1). If when $\epsilon=0, \lambda=\lambda_{0}$ is a simple eigenvalue with the eigenfunction satisfying (4.5), then

$$
E_{\epsilon}\left(\lambda_{0}, 0\right)=\frac{1}{\hat{c}}\left[a_{1} b_{1} \frac{p_{1}(-L)}{p_{0}(-L)^{2}}-\hat{c}^{2} a_{2} b_{2} \frac{p_{1}(L)}{p_{0}(L)^{2}}-\int_{-L}^{L} p_{1}(s) \frac{\partial y_{0}}{\partial x}(s)^{2}+\left(q_{1}(s)-\lambda_{0} w_{1}(s)\right) y_{0}(s)^{2} \mathrm{~d} s\right] .
$$

Proof: The system (2.5) is

$$
\frac{\partial}{\partial x} \boldsymbol{\Phi}=\boldsymbol{A}(x ; \lambda, \epsilon) \boldsymbol{\Phi}, \quad \boldsymbol{\Phi}(-L ; \lambda, \epsilon)=\left(\begin{array}{cc}
-b_{1} / p(-L) & c a_{1} \\
a_{1} & c b_{1} / p(-L)
\end{array}\right),
$$

where the constant $c$ is chosen so that $\operatorname{det}(\boldsymbol{\Phi}(-L ; \lambda, 0))=1$, i.e.,

$$
c=-\frac{1}{\left(b_{1} / p_{0}(-L)\right)^{2}+a_{1}^{2}} \text {. }
$$

Using the fact that

$$
\frac{1}{p}=\frac{1}{p_{0}} \frac{1}{1+\epsilon p_{1} / p_{0}}=\frac{1}{p_{0}}\left(1-\epsilon \frac{p_{1}}{p_{0}}+\mathcal{O}\left(\epsilon^{2}\right)\right),
$$

we have the Taylor expansions

$$
\boldsymbol{A}(x ; \lambda, \epsilon)=\left(\begin{array}{cc}
0 & 1 / p_{0} \\
q_{0}-\lambda w_{0} & 0
\end{array}\right)+\epsilon\left(\begin{array}{cc}
0 & -p_{1} / p_{0}^{2} \\
q_{1}-\lambda w_{1} & 0
\end{array}\right)+\mathcal{O}\left(\epsilon^{2}\right),
$$

and

$$
\boldsymbol{\Phi}(-L ; \lambda, \epsilon)=\left(\begin{array}{cc}
-b_{1} / p_{0}(-L) & c a_{1} \\
a_{1} & c b_{1} / p_{0}(-L)
\end{array}\right)+\epsilon \frac{b_{1} p_{1}(-L)}{p_{0}(-L)^{2}}\left(\begin{array}{cc}
1 & 0 \\
0 & -c
\end{array}\right)+\mathcal{O}\left(\epsilon^{2}\right) .
$$

Letting $\boldsymbol{\Psi}(x)=\boldsymbol{\Phi}_{\epsilon}\left(x ; \lambda_{0}, 0\right)$, where $\lambda_{0}$ is an eigenvalue, we have that $\boldsymbol{\Psi}$ solves the system

$$
\frac{\partial}{\partial x} \boldsymbol{\Psi}=\boldsymbol{A}\left(x ; \lambda_{0}, 0\right) \boldsymbol{\Psi}+\boldsymbol{A}_{\epsilon}(x ; \lambda, 0) \boldsymbol{\Phi}\left(x ; \lambda_{0}, 0\right), \quad \boldsymbol{\Psi}(-L)=\boldsymbol{\Phi}_{\epsilon}\left(-L ; \lambda_{0}, 0\right),
$$

which by variation of parameters has the solution

$$
\boldsymbol{\Psi}(x)=\boldsymbol{\Phi}\left(x ; \lambda_{0}, 0\right) \boldsymbol{\Phi}\left(-L ; \lambda_{0}, 0\right)^{-1} \boldsymbol{\Phi}_{\epsilon}\left(-L ; \lambda_{0}, 0\right)+\boldsymbol{\Phi}\left(x ; \lambda_{0}, 0\right) \int_{-L}^{x} \boldsymbol{\Phi}\left(s ; \lambda_{0}, 0\right)^{-1} \boldsymbol{A}_{\epsilon}(s ; \lambda, 0) \boldsymbol{\Phi}\left(s ; \lambda_{0}, 0\right) \mathrm{d} s .
$$

The Evans function is given by

$$
E(\lambda, \epsilon)=\operatorname{det}\left(\begin{array}{c}
\boldsymbol{\Phi}(L ; \lambda, \epsilon)\left(\begin{array}{l}
1 \\
0
\end{array}\right) \\
-b_{2} / p(L) \\
a_{2}
\end{array}\right),
$$

which by the product rule yields that its partial derivative with respect to $\epsilon$ is

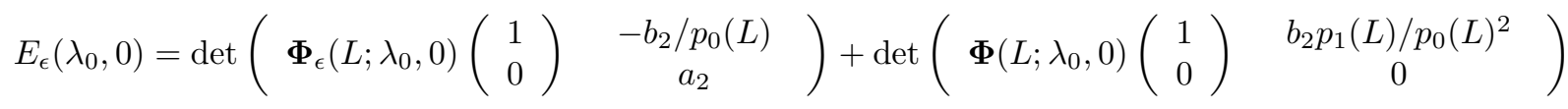

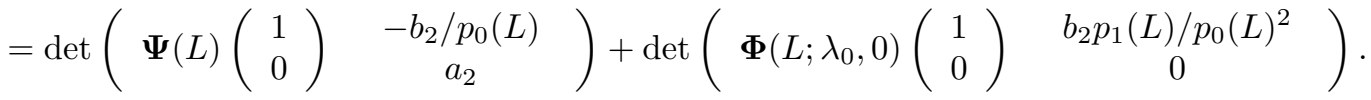

From (4.4) we have that

$$
\left(\begin{array}{c}
-b_{2} / p(L) \\
a_{2}
\end{array}\right)=\frac{1}{\hat{c}} \Phi\left(L ; \lambda_{0}, 0\right)\left(\begin{array}{l}
1 \\
0
\end{array}\right)
$$


which upon using the fact that $\operatorname{det} \boldsymbol{\Phi}\left(L ; \lambda_{0}, 0\right)=1$ gives

$$
\begin{gathered}
\operatorname{det}\left(\boldsymbol{\Psi}(L)\left(\begin{array}{l}
1 \\
0
\end{array}\right) \begin{array}{c}
-b_{2} / p_{0}(L) \\
a_{2}
\end{array}\right)=\frac{1}{\hat{c}}\left[\operatorname{det}\left(\boldsymbol{\Phi}\left(-L ; \lambda_{0}, 0\right)^{-1} \boldsymbol{\Phi}_{\epsilon}\left(-L ; \lambda_{0}, 0\right)\left(\begin{array}{l}
1 \\
0
\end{array}\right) \begin{array}{c}
1 \\
0
\end{array}\right)+\right. \\
\left.\operatorname{det}\left(\int_{-L}^{L} \boldsymbol{\Phi}\left(s ; \lambda_{0}, 0\right)^{-1} \boldsymbol{A}_{\epsilon}\left(s ; \lambda_{0}, 0\right) \boldsymbol{\Phi}\left(s ; \lambda_{0}, 0\right)\left(\begin{array}{c}
1 \\
0
\end{array}\right) \text { d } s \quad \begin{array}{c}
1 \\
0
\end{array}\right)\right]
\end{gathered}
$$

Using (4.7) and simplifying gives

$$
\operatorname{det}\left(\boldsymbol{\Phi}\left(-L ; \lambda_{0}, 0\right)^{-1} \boldsymbol{\Phi}_{\epsilon}\left(-L ; \lambda_{0}, 0\right)\left(\begin{array}{l}
1 \\
0
\end{array}\right) \quad \begin{array}{l}
1 \\
0
\end{array}\right)=a_{1} b_{1} \frac{p_{1}(-L)}{p_{0}(L)^{2}}
$$

while using (4.6) and simplifying gives

$$
\begin{aligned}
& \operatorname{det}\left(\int_{-L}^{L} \boldsymbol{\Phi}\left(s ; \lambda_{0}, 0\right)^{-1} \boldsymbol{A}_{\epsilon}\left(s ; \lambda_{0}, 0\right) \boldsymbol{\Phi}\left(s ; \lambda_{0}, 0\right)\left(\begin{array}{l}
1 \\
0
\end{array}\right) \mathrm{d} s \quad \begin{array}{l}
1 \\
0
\end{array}\right)= \\
& -\int_{-L}^{L} p_{1}(s) \frac{\partial y_{0}}{\partial x}(s)^{2}+\left(q_{1}(s)-\lambda_{0} w_{1}(s)\right) y_{0}(s)^{2} \mathrm{~d} s .
\end{aligned}
$$

Consequently,

$$
\operatorname{det}\left(\boldsymbol{\Psi}(L)\left(\begin{array}{c}
1 \\
0
\end{array}\right) \begin{array}{c}
-b_{2} / p_{0}(L) \\
a_{2}
\end{array}\right)=\frac{1}{\hat{c}}\left[a_{1} b_{1} \frac{p_{1}(-L)}{p_{0}(L)^{2}}-\int_{-L}^{L} p_{1}(s) y_{0}^{\prime}(s)^{2}+\left(q_{1}(s)-\lambda_{0} w_{1}(s)\right) y_{0}(s)^{2} \mathrm{~d} s\right] .
$$

Upon using (4.5) we have

$$
\operatorname{det}\left(\begin{array}{cc}
\boldsymbol{\Phi}\left(L ; \lambda_{0}, 0\right) & \left(\begin{array}{c}
1 \\
0
\end{array}\right) \\
b_{2} p_{1}(L) / p_{0}(L)^{2} \\
0
\end{array}\right)=-\hat{c} a_{2} b_{2} \frac{p_{1}(L)}{p_{0}(L)^{2}} .
$$

Combining the above equalities gives the final result.

In a particular case where $p_{1}=w_{1} \equiv 0$, the expression for $E_{\epsilon}\left(\lambda_{0}, 0\right)$ greatly simplifies to

$$
E_{\epsilon}\left(\lambda_{0}, 0\right)=-\frac{1}{\hat{c}} \int_{-L}^{L} q_{1}(s) y_{0}(s)^{2} \mathrm{~d} s .
$$

Upon using the expansion (4.2) with the $\lambda$-derivative expression of (4.3), we get the following (simplified) result concerning the movement of eigenvalues.

cor:41 Corollary 4.2. Suppose that the coefficients in (4.2) satisfy $p_{1}=w_{1} \equiv 0$. If when $\epsilon=0, \lambda=\lambda_{0}$ is a simple eigenvalue whose eigenfunction satisfies (4.5), then the eigenvalue has the expansion

$$
\lambda(\epsilon)=\lambda_{0}+\left(\frac{\int_{-L}^{L} q_{1}(s) y_{0}(s)^{2} \mathrm{~d} s}{\int_{-L}^{L} w_{0}(s) y_{0}(s)^{2} \mathrm{~d} s}\right) \epsilon+\mathcal{O}\left(\epsilon^{2}\right) .
$$

For a concrete example, suppose that

$$
q_{1}(x)=\delta(x+\rho)+\delta(x-\rho), \quad 0<\rho<L
$$

(compare to (3.6)). Suppose further that $a_{1}=a_{2}, b_{1}=b_{2}$ and $p_{0}, q_{0}, w_{0}$ satisfy the hypotheses of Theorem 2.8. The Evans function is then conjugate symmetric, and all of the eigenvalues for the Sturm-Liouville problem come in complex-conjugate pairs for all $\epsilon$. Assume that when $\epsilon=0, \lambda=\lambda_{0}$ is a simple real-valued zero of the Evans function. If the associated eigenfunction is denoted by $y_{0}(x)$, upon using the fact that

$$
\int_{-L}^{L} q_{1}(x) y_{0}(s)^{2} \mathrm{~d} s=y_{0}(-\rho)^{2}+y_{0}(\rho)^{2}
$$


we have by Corollary 4.2 that the eigenvalue moves like

$$
\lambda(\epsilon)=\lambda_{0}+\left(\frac{y_{0}(-\rho)^{2}+y_{0}(\rho)^{2}}{\int_{-L}^{L} w_{0}(s) y_{0}(s)^{2} \mathrm{~d} s}\right) \epsilon+\mathcal{O}\left(\epsilon^{2}\right) .
$$

This expression can be further simplified. In Lemma 2.7 we saw that

$$
y_{0}(-\rho)=\overline{y_{0}(\rho)}
$$

hence,

$$
y_{0}(-\rho)^{2}+y_{0}(\rho)^{2}=y_{0}(\rho)^{2}+{\overline{y_{0}(\rho)}}^{2}=2 \operatorname{Re}\left(y_{0}(\rho)^{2}\right) .
$$

Since the zero will remain real-valued for (at least) small $\epsilon$, it must be true that

$$
\operatorname{Im}\left(\frac{y_{0}(-\rho)^{2}+y_{0}(\rho)^{2}}{\int_{-L}^{L} w_{0}(s) y_{0}(s)^{2} \mathrm{~d} s}\right)=0 .
$$

Since we just computed that the numerator is real-valued, this necessarily implies that the denominator is also real-valued. In conclusion, we have that the eigenvalue moves like

$$
\lambda(\epsilon)=\lambda_{0}+\frac{2 \operatorname{Re}\left(y_{0}(\rho)^{2}\right)}{\int_{-L}^{L} w_{0}(s) y_{0}(s)^{2} \mathrm{~d} s} \epsilon+\mathcal{O}\left(\epsilon^{2}\right) .
$$

For the example discussed in Section 3.1, where $p_{0}=w_{0} \equiv 1$ and $q_{0} \equiv 0$, and the boundary conditions have $a_{1}=a_{2}=1, b_{1}=b_{2}=0$, the eigenvalues and associated eigenfunctions are given by

$$
\lambda=\frac{\pi^{2} n^{2}}{4 L^{2}}, \quad y(x)=\sin \left(\frac{\pi n}{2 L} x\right) .
$$

In this case (4.8) becomes for each $n=1,2, \ldots$,

$$
\lambda=\frac{\pi^{2} n^{2}}{4 L^{2}}+\frac{2}{L} \sin ^{2}\left(\frac{\pi n \rho}{2 L}\right) \epsilon+\mathcal{O}\left(\epsilon^{2}\right) .
$$

\section{REFERENCES}

ardner:sis91

berman: apd04

lathews : caf06

limani:osi08

nagle:fod00

[1] R. Gardner and C.K.R.T. Jones. A stability index for steady state solutions of boundary value problems for parabolic systems. J. Diff. Eq., 91:181-203, 1991.

[2] R. Haberman. Applied Partial Differential Equations with Fourier Series and Bounday Value Problems. Pearson Education, Inc., Fourth edition, 2004.

[3] J. Mathews and R. Howell. Complex Analysis for Mathematics and Engineering. Jones and Bartlett Publishers, Fifth edition, 2006.

[4] Z. Musslimani, K. Makris, R. El-Ganainy, and D. Christodoulides. Optical solitons in $\mathcal{P} \mathcal{T}$ periodic potentials. Phys. Rev. Lett., 100:030402, 2008.

[5] R. Nagle, E. Saff, and A. Snider. Fundamentals of Differential Equations. Addison-Wesley, Fifth edition, 2000. 